in: D. Heyer \& R. Mausfeld (eds.) (2002). Perception and the Physical World. Chichester: Wiley

\title{
The Physicalistic Trap in Perception Theory
}

\author{
Rainer Mausfeld
}

\section{Table of Contents}

Introduction

- The physicalistic trap in elementaristic approaches to perception

- Example I: The problem of colour constancy

- Example II: Are there perceptual errors and illusions?

- Example III: The preoccupation with linear perspective in theories of picture perception

- The physicalistic trap in functionalist and computational approaches to perception

- Example IV: Bayesian approaches to perception

- Example V: Colour constancy from a computational point of view

- Example VI: Evolutionary internalized regularities of the physical world

- Perception theory beyond the physicalistic trap

- Appendix: Historical annotations on the notions of sensory data-based computation vs. triggering in perception theory

- References 


\section{Introduction}

It is a well-known observation from the history of the natural sciences that any field that is not yet mature enough to have a proprietary and sufficiently rich theoretical core of its own has to face the problem of which concepts and notions, and thus which theoretical language, to chose for the segmentation and theoretical organization of relevant phenomena. Perceptual psychology is no exception in this regard. The lack of an appropriate theoretical language of its own results in the temptation to borrow theoretical structure from neighbouring disciplines and to use as a surrogate a theoretical structure that has been developed for different explanatory purposes (and thus is alien to the intrinsic structure of the field in question). In perception theory the fields from which these surrogate structures have been borrowed are sensory physiology and physics. In this essay I will be concerned with what may be called the physicalistic trap, namely misconceptions in perception theory that are based on the idea of slicing the nature of perception along the joints of physics and on corresponding ill-conceived 'purposes' and 'goals' of the perceptual system. Corresponding misconceptions have been disclosed ever since the beginnings of perception theory, in particular by the Gestaltists. Current developments, however, have largely fallen back upon these misconceptions, albeit on levels of considerable technical sophistication. Nevertheless, there is, with respect to the principal points of concern, hardly anything new to say and so I resign myself to assembling a few reminders. My primary concern, however, is not a critique of these misconceptions; rather I intend to delineate, using the physicalistic trap as a background for confrontation, a line of inquiry, inspired by ethology, that helps to focus attention on problems that a genuine theory of perception has to deal with and that seem to me to be largely ignored in current approaches.

Perceptual psychology, understood as the endeavour to theoretically understand, within the conceptual framework of the natural sciences, certain aspects of mental activity, has to develop, just as other domains of the natural sciences did, an autonomous theoretical language that appropriately reflects the internal structure of perception and is sufficiently rich to account in an explanatorily satisfactory way for what are considered to be basic principles and phenomena of perception. Perceptual psychology aims at a proper theoretical understanding of the structure of those mental representations that refer to our interacting with the environment. Its theoretical language, therefore, hinges upon how we describe biologically relevant aspects of the environment of the organism - which I will briefly refer to as a description of the 'external world' -, and how we relate such a description to a 
theoretical description of the internal structure of perception. That such a description depends on the structure of the sensory system under scrutiny is obvious and almost trivial in cases where we are dealing with sensory receptors and their transduction properties. Different biological species exhibit quite different ways of parsing the physical energy that hits upon the organism into sense modalities and perceptual qualities. Most of the spatio-temporal pattern of this energy is not used for biological purposes (we cannot sense e.g. the direction of magnetic fields). Only some aspects are filtered out for the specific purposes of a perceptual system and transduced into a neural code, whose properties no longer allow the specific physical properties it was caused by to be identified. With respect to transduction properties it is obvious that the choice of concepts that are considered appropriate for a description of properties of the external world (e.g. photons in the case of photoreceptors) is determined by structural properties of the perceptual system. It is less obvious, though indisputable, that an appropriate, i.e. theoretically fruitful, description of the 'external world' crucially depends also on more abstract representational properties of the perceptual system. Because we are still far from having a satisfactory theoretical understanding of those abstract properties we can rely only on our best current understanding in choosing appropriate concepts for describing relevant properties of the external world and of the sensory input - a problem of great concern for, e.g., the Gestaltists, Gibson, Marr and Shepard. The development of a conceptual vocabulary for describing, in a theoretically fruitful way, perceptually relevant aspects of the external world is a prerequisite of perception theory. However, the structure of perceptual representations does not simply mirror and is not solely moulded by properties of the external world but rather co-determined by internal aspects, such as internal functional constraints or internal architectural constraints. Perceptual representations must not only be adequate with respect to the external world; they must also be functionally adequate, i.e. they have to fit into the entire perceptual architecture including its two fundamental interfaces, viz. the interface with the motor system and that with the cognitive system. These internal aspects have been largely disregarded in current perception theory. Fundamental concepts of perception theory and ideas about internal representations have instead been derived from considerations that are alien to the intrinsic structure of perception. Physics and sensory physiology have served as sources from which core theoretical notions of perception theory have been surreptitiously borrowed. In describing certain lines of thinking as a physicalistic trap, it is not my intention to denigrate their role in perceptual research. It is indisputable that ideas associated with the physicalistic trap are an indispensable part of our ordinary and metatheoretical talk about perception and can, in principle, serve as heuristics in perceptual research. They become a trap, however, when they result in a tendency to dodge an essential task of perceptual research, viz. the identification of the internal conceptual structure of perception. Characteristic theoretical distortions result from using structural elements that serve the explanatory needs of other domains of inquiry as inappropriate surrogates for the yet-to-be 
discovered structural elements on which explanatory accounts of perceptual representations have to be based. I will illustrate these distortions by discussing six examples in some detail. However, pointing out these distortions is not meant as an end in itself. Rather I intent to use them as whetstones, as it were, to bring out questions and problems that are largely disregarded in current research but that seem to me to be at the core of any genuine theory of perception.

I will only mention in passing, in this introduction, misconceptions based on deriving basic elements of the theoretical structure of perception theory from sensory physiology. Due to the pre-theoretical attribution of certain classes of sensations to sensory organs, it is quite natural from a heuristic point of view that the sense modalities have been considered both as the natural starting point and as the natural units of analyses for perceptual psychology. Thus it was held that they could to a large extent be treated in isolation. However, such a theoretical segmentation according to the classification of sensory input channels almost certainly impedes a deeper theoretical understanding of the intrinsic structure of perception because it distracts the theoretical focus from what can be considered one of the core characteristics of perceptual representations: The internal structure of perception is determined in an essential manner by transmodal or amodal representations of the physico-biological environment, and is thus not reducible to the structure of isolated input channels. ${ }^{1}$ The ability to free itself from the properties of specific sensory input channels, i.e. from the properties of sensory organs and the neural processes that mediate between the sensory organs and the perceptual representations, is a remarkable achievement of the perceptual system. The sensory input channels only serve as a perceptual medium whose idiosyncratic coding properties have to be discounted by the perceptual system in transmodal representations (in terms of its representational primitives) of the physico-biological environment.

In confusing properties of the transmission medium with internal properties of the perceptual system, variants of the physiologistic trap in perception theory derive alleged 'stages of perception' from stages of neurophysiological processing. In this regard the distinction between 'high level' vs. 'low level' processes has been particularly influential in recent years. This distinction is usually assumed to refer to some kinds of fixed and empirically given states of the system under scrutiny. However, without a specific theory about perceptual processes no criteria for such a distinction exist. ${ }^{2}$ Even in neurophysiology the high amount of vertical interconnectivity deprives this distinction of any precise meaning (unless we derive its meaning from and relative to a specific model). In perception theory

\footnotetext{
${ }^{1}$ Interesting new kinds of relevant evidence come from experiments with babies and very young children (e.g. Streri, Spelke \& Rameix, 1993).

${ }^{2}$ Of course, in anatomy we can speak - though in a theoretically not very interesting way - of 'low levels' or 'high levels' of the visual system, as we can in machine vision, where these levels mirror the way we have designed a system.
} 
such distinctions, if they can be specified at all, become entirely theory-dependent. Here, absolute high-low level distinctions based on neurophysiological investigations of neural structure erroneously take properties of the medium as theoretical building blocks for explaining perceptual representations. The physiologistic trap thus slices the nature of perception according to the physiological nature of the sense modalities and essentially conceives of perception as consecutive transformations of the sensory input.

The physicalistic trap comes in various guises and is sometimes difficult to disclose. Variants of it have pervaded theoretical attempts to understand the nature of perception ever since the beginnings of systematic inquiries into the nature of perception. Examples can be found under headings as diverse as

- the concept of perceptual illusion

- the idea of a pre-theoretical segregation of phenomena into primary and contextual ones

- the idea that the structure of perception is based on internal correlates of physical scales

- the inverse optics approach of recovering properties of the world from image structure

- Bayesian approaches to perception that base their physical description of the sensory input on categories of the yet-to-be identified and explained perceptual output.

We can distinguish two different varieties of the physicalistic trap: one that derives theoretical descriptions of the structure of perception from descriptions of the sensory input in terms of elementary physical variables, ${ }^{3}$ and one that derives them from descriptions of the distal stimulus in terms of concepts from folk physics and physical common sense taxonomies, such as surface, object, illumination, shadow, etc. I will address both in turn and illustrate them by way of examples that seem to me particularly instructive in this respect.

\footnotetext{
${ }^{3}$ The physicalistic trap bears some relation to what, in classical psychophysics, Titchener called the "stimulus error", by which he meant problems that result from confusing mental aspects with aspects of the stimuli that give rise to them (see Boring, 1921).
} 


\section{The physicalistic trap in elementaristic approaches to perception}

The physicalistic trap in elementaristic approaches to perception looms up most clearly in what may be called the measurement device conception of perception. This is an influential though highly misleading heuristics that comes in many guises and is rarely spelled out explicitly. It is based on the metaphor of conceiving the perceptual system as some kind of measurement device that has to inform us about elementary physical quantities. Being a legacy of the way we have separated physical and psychological aspects in the philosophical history of the field since the time of the pre-Socratics this metaphor has governed our thinking in the study of perception ever since. Along with this conception comes the idea that there are atoms of perception, as it were, that are strongly tied to these elementary physical variables: namely the sensations from which perceptions - as something referring to the distal external world - are constructed. According to this view the energy pattern from the external world that affects the senses is evaluated by the perceptual system locally, as it were, and leads to 'sensations' out of which 'higher level perceptions' are constructed. Elementary physical variables, like energy of sound, intensity and wavelength of light, are regarded as the basis for fundamental perceptual variables, like loudness, brightness and colour. ${ }^{4}$

The measurement device conception considers those perceptual phenomena for which a stable correspondence between local properties of the physical stimulus and the perceptual appearance or neural reaction can be observed to be the basic and fundamental ones. 'Normal perception', then, can be disturbed or biased, by so-called 'context effects'. The price that had to be paid for this way of slicing up the space of phenomena into 'basic' and 'secondary' ones was that one had to classify phenomena that are fundamental with respect to the representational primitives of the system inappropriately as 'context effects', 'perceptual illusions', 'constancy phenomena', or 'higher order phenomena'. The problem of colour constancy may serve to illustrate the ensuing theoretical distortions.

\footnotetext{
${ }^{4}$ This perspective is most explicit in the research programme of classical psychophysics. Stevens based his approach on a reference to elementary variables of physics, like length, weight, light intensity, or frequency of sound waves, and tried to construct for each of these variables a transformation that characterizes its subjective analogue. The perceptual system is conceived as some kind of measuring device for the textbook variables of physics. A theoretically more sophisticated form is the research perspective of R.D. Luce, who tried to establish a structure of interrelated subjective scales that allow a reduction to a simple pattern of units and simple connections with the scales of physics: an internal analogue of the dimensional structure of physics.
} 


\section{Example I: The problem of colour constancy}

The measurement device misconception has a long tradition in the field of colour perception. ${ }^{5}$ It is disguised here in the idea that there are some kinds of 'raw colours' or 'primordinal colours' that are given by the receptor excitations elicited by the local incoming light stimulus and that are transformed and modified in subsequent stages of processing in order to fulfil certain requirements, such as sensitivity regulations, optimal and efficient coding or invariance requirements. In the wake of these approaches it became a matter of course to conceive decontextualized small colour patches (that virtually have no localisation or orientation) - such as the ones underlying CIE colour space - as the building blocks of colour perceptions, as raw and pure colour sensations or unanalysable instantiations of colour qualia. The local connection between these 'primordinal colours' and colour appearances, as expressed for example in CIE space, is considered as the 'normal case' and thus the so-called constancy phenomena are regarded as more surprising and in greater need of explanation than the 'normal case'. Perceptual representations of, say surface colours, are, on this view, built up in a locally-atomistic way from these raw colours, i.e. the perceptual bases of colour perception are the primary colour codes that arise from the transduction of physical energy into neural codes and that are transformed by 'secondary' and 'higher' processes into representations of, say, surface colours. In other words, the 'primary elements' of colour perception are constituted on the level on which a stable correspondence between local properties of the sensory input and the neural reaction can be observed, and are then further processed and transformed, modified, or supplemented by 'secondary', 'higher order' processes to yield perceptual achievements or appearances. By focussing on transformations and 'secondary' operations of primary colour codes the elementaristic perspectives in colour research divorced colour from its intrinsic anchorage within the perceptual architecture. The theoretical treatment of simultaneous contrast phenomena in centre-surround situations provides an interesting case in point of how classifications based on a distinction between elementaristic primary colour codes and 'context effects' can produce taxonomic artefacts that veil core structural principles underlying colour perception. Many classic writers realized that phenomena associated with the geometrical configuration of small and sharply demarcated infields in large surrounds cannot appropriately be accounted for by simple transformations of elementary colour codes but require explanations based on the internal concepts of 'surface colour' and 'illumination colour'. For instance, Bühler (1922, p. 131) interpreted the phenomenon of simultaneous contrast in such situations as a degenerate marginal phenomenon attesting to the visual system's

\footnotetext{
${ }^{5}$ A variant of the measurement device concept is what Gilchrist called, with respect to brightness perception, the photometer metaphor (Gilchrist, 1994).
} 
capability of preserving colours under changes of illumination. The perceptually relevant aspect of such situations is the figure-ground segmentation that they give rise to. Rubin (1921, p. 56), for instance, observed that transformations in the direction of colour constancy are stronger when a certain area is perceived as figure rather than as ground. Empirical and theoretical evidence (cf. Mausfeld \& Niederée, 1993; Mausfeld, 1998) suggests regarding centre-surround type configurations as 'minimal' stimuli, as it were, for triggering, albeit in rudimentary form, representations that are based on the representational primitives 'object colour' and 'illumination colour'.

Though the elementaristic perspective has proved to be fruitful for the neurophysiological purposes of understanding the nature of transduction and peripheral coding, it is at a cost for perceptual psychology, of which both Helmholtz and Hering were well aware. They realized that concepts from sensory physiology alone do not constitute an appropriately rich theoretical language for dealing with perception but that a richer set of concepts, including "unconscious inferences" in the case of Helmholtz, and "memory colours" in the case of Hering, is required for appropriate explanatory frameworks. It is not the specific nature of such concepts themselves that is of interest here, but rather the unequivocal elucidation that an additional level of analysis beyond the neurophysiological one - and correspondingly a different theoretical language - is needed for a successful theoretical account of perception. ${ }^{6}$ Though introducing 'psychological' concepts as an additional level of analysis increased the explanatory power of the theoretical language it accentuated and deepened inappropriate classifications of perceptual phenomena into 'basic' and 'secondary' ones.

The problem of colour constancy is the legacy of the way the structure of perception has been divided into foundational building blocks of 'raw sensations' that exhibit a stable correspondence to their local physical causes, on the one hand, and 'higher order', 'psychological' effects that connect these 'raw sensations' with biologically meaningful distal objects, on the other hand. The field of colour perception, more than other fields of perception, is dominated by variants of the measurement device misconception. ${ }^{7}$ The problem of colour constancy came to be regarded as a problem confined to 'pure' colour perception, where transformations of some 'raw colours' result in a discounting of the illuminant. As a result of this way of trivializing the problem of the internal representational structure of colour perception and of idealizing away the perception of the illumination the problem of colour constancy became misidealized and

\footnotetext{
${ }^{6}$ These insights, as right and important as they were, have encumbered further developments with an unfortunate and misleading cleavage of perception into 'physiological' and 'psychological' processes.

${ }^{7}$ This may be exemplified by Buchsbaum \& Gottschalk's (1983, p.92) remark that "The visual system is concerned with estimating the spectral functional shape of the incoming colour stimulus."
} 
misrepresented. ${ }^{8}$ Gelb (1929) was the first to arrive at a clear understanding of the structure of the problem of colour constancy. He considered the distinction between 'physiological' and 'psychological' levels as "wrong" and regarded, in Gelb (1932), any such "dualisms of explanatory principles" as inappropriate and misleading. He convincingly argued "that the problem of colour constancy, rather than being a problem of an alleged discrepancy between 'stimulus' and 'perceived colour', has to do with the general problem of the constitution and structure of our perceptual visual world. The phenomenal segregation into illumination and illuminated object (i.e. the correlate of the percept 'object colour') reveals a propensity of our sensorium and is nothing but the expression of a certain structural form of our perceptual visual world." (Gelb, 1929, p. 672) In the same vein, Cassirer (1929, p. 155) considers the phenomena relating to a dual account of an 'object colour' and an 'illumination colour' attached to it not to be a result of some additional processing, but rather as an expression of the "very primordial format of organization". Such insights into the structural role of colour within perceptual representations were far from being mere speculations, but rather were, even at that time, strongly suggested by the theoretical and empirical evidence available. Yet they have been almost completely ignored in subsequent approaches. I will return to these issues below in the discussion of computational treatments of colour constancy.

\section{Example II: Are there perceptual errors and illusions?}

Since the time of the pre-Socratics, who were the first to deal with the 'fallibility' of the senses, there has been a long history of wrestling with the concept of perceptual errors, both in epistemology and in perceptual psychology. Historically, the enterprise of physics began when in pre-Socratic times a distrust in the senses showed up, which basically stemmed from the observation that different senses can lead us to different beliefs about the world (think of a rod half dipped in water). The attempt to accomplish a picture of the 'real world' behind the 'unreliable picture' that our senses convey to us is the starting point of physics. Since then we have a split between the world as experienced and the world as pictured by physics. We began to take physics as a reference system for the veridicality of our perceptions and to speak of perceptual illusions when we became aware of a discrepancy between the physical description and our perception.

\footnotetext{
${ }^{8}$ It was in this form that it was taken up as the computational problem of arriving, on the basis of the sensory input, at colour designators of a scene that are invariant under different illumination (see Example V). Though great conceptual advances and insights with respect to the development of an ecological physics have been brought forth by corresponding investigations, they also contributed to veiling the proper structure of the perceptual problem involved.
} 
There are many intricate philosophical issues connected with this issue. Fortunately, however, most of these have no bearing on the usage of the concept of 'error' in visual psychophysics, which I will address here. Particularly, I can leave aside all issues centring around the (legitimate) concept of 'error of perceptual judgement' (which every-day usages refer to).

At the root of the idea of perceptual errors - again: in the sense of 'errors of perceptual mechanisms', not as errors of perceptual judgement - is the conviction that the goal of the visual apparatus is something like a veridical seeing of the true physical situation. But what is the 'true physical situation'? What is the reference frame for the beliefs and expectations that give rise to a distinction between 'normal' and 'illusionary' perception?

Most often, our expectations derive from folk physics: perception is called veridical if it conforms to a folk-physics description of what is out there. There is, of course, no good reason why such expectations should be relevant for guiding perceptual research (though, they served historically as heuristic starting-points that brought basic problems of perceptual research to our attention). A perceptual phenomenon is not just therefore in need of explanation because it does not conform to some pre-theoretical expectations. Folk-physical descriptions, however, often receive a higher epistemological dignity, as it were, and when there is a conflict between two levels of description of what is out there - including conflicts between descriptions provided by different sense modalities like touch and sight, or vision and static sense -, perception is considered as illusionary. Often such expectations about the alleged correct output of visual mechanisms go hand in hand with the idea that the goal of the perceptual system is to 'inform' us about the true physical situation as measured by elementary physical devices, i.e. with the measurement device conception of perception. ${ }^{9}$

Once we retreat from folk physics as a reference frame guiding our expectations about perception, our expectations about what has to be considered as accidental or essential, as proper or improper, as normal or deviant can only be derived from specific theoretical ideas about the perceptual system. If we had a complete understanding of the visual system, we would certainly cease to call certain responses 'errors'. Of course, we can speak of non-optimality of design in terms of

\footnotetext{
${ }^{9}$ Whether we call a phenomenon an illusion depends on how sophisticated our ideas about the properties of internal coding are. The poorer the theoretical structure the greater the tendency is to guide expectations by a measurement device conception of perception. This can be illustrated by comparing the Müller-Lyer demonstration with Wallach's brightness demonstrations that underly the formulation of his ratio principle. The Wallach demonstrations refer to a situation that has, with respect to brightness, the same logical structure that the Müller-Lyer demonstration has with respect to length. While the Müller-Lyer demonstration is called an illusion, no one would, however, refer to the Wallach demonstration as a brightness illusion, which is rather considered as demonstrating a relational coding property of brightness.
} 
an adaptational coupling, or malfunctioning in the clinical sense, but these are different concepts.

The locution, however, that the perceptual system is susceptible to illusions under ecologically atypical conditions requires us to go beyond perception theory proper and to state what function the system is serving and what the proper function of the system is. Take the example of the frog's 'fly detector', i.e. that part of the frog's visual system whose proper function, according to the frog's specific evolutionary history, is the detection of flies. This mechanism will still perform its adaptive function in an environment in which no flies exist but instead a species 'pellies', which are nutritious small dark moving pellets. In that case the frog's visual system does not refer to flies any longer and thus fails to perform its proper function. A generation of scientists unaware of this change of environment would characterize this visual submechanism as a pellies-detector. ${ }^{10}$ This may illustrate why, among other reasons, I prefer to restrict the domain of a formal theory of perception to an explanatory account of the perceptual system of a given organism in terms of its internal functioning, and to shift talk about evolutionary history and proper function to a metatheoretical level. Notions like 'perceptual error', 'veridicality', 'reference' or 'proper function' do not figure in a formal theory of perception. They only come into play if we, in ordinary discourse or as scientists from a metatheoretical viewpoint, make a conjecture about the 'proper' object of perception and the 'true' antecedents of the sensory information, among the infinite set of potential causal antecedents. No such notions can enter our descriptions of the functioning of perceptual subsystems of the mind. The visual system simply does what it does. There are no 'errors' with respect to its representational primitives, an idea already lucidly expressed by Helmholtz (1855, p. 100): "The senses cannot deceive us, they work according to their established immutable laws and cannot do otherwise. It is us who are mistaken in our apprehension of the sensory perception."

\section{Example III: The preoccupation with linear perspective in theories of picture perception}

Pictures and pictorial representations can, from a naive point of view, also be regarded as a kind of illusion because they evoke perceptual impressions of objects, spatial relations or events in us that are actually not there. Above all

\footnotetext{
${ }^{10}$ Dretske's (1986) discussion of certain magnetotactic marine bacteria is also instructive in this respect. These bacteria use a physically contingent relation between geomagnetic north and oxygen-free water that holds in the northern hemisphere to orient themselves away from deadly oxygen-rich surface water (Blakemore \& Frankel, 1981). Transplanting this kind of bacterium to the southern hemisphere results in a deadly 'perceptual error', though "the magnetotactic system functions as it is supposed to function, as it was (presumably) evolved to function."
} 
because of this, phenomena that can be encountered in picture perception (cf. Hochberg, 1980; Gombrich, 1982; see also Schwartz, this volume) have often been regarded as particularly challenging for theories of visual perception. This is surprising because picture perception hardly constitutes a natural domain for exploring core structural elements of perception. Like all perceptual tasks that involve human artefacts, it rests on and exploits the complex interactions of given perceptual structures and interpretative faculties, whose properties are presently only poorly understood. Artefacts depend on human intentions and their use therefore is subject to interpretation; this holds for TV screens, microscopes, books or pictures. They exploit given capacities, but one has to understand what they were designed for. From the perspective of the cognitive sciences, picture perception does not constitute a domain of phenomena that is bound together by some specific set of explanatory principles. Nonetheless, picture perception has become a field of inquiry within perceptual psychology that has brought forth its own lines and frameworks of inquiry and its own specific problems. A dominant theme in the field of picture perception have been issues centring around notions of perceptual space and the extent to which corresponding percepts can be evoked by features of pictorial representations, notably by linear perspective (cf. Haber, 1980; Rogers, 1995).

\section{Linear Perspective as an artist's means to achieve 'visual truth' in paintings}

The interest in techniques of linear perspective arose during the Renaissance and was motivated by the artists' desires to imitate nature and to achieve 'visual truth' in their paintings. This gave rise to corresponding inquiries into artistic techniques for the evocation of space and in particular into techniques how to create on a canvas geometrically correct two-dimensional pictorial representations of the three-dimensional layout of the pictured scene. In these investigations, as Kemp (1990, p.165) has observed, "the eye figures little, the mind features even less." Rather what was to be accomplished was "the demonstration of an internally consistent system of the spatial elements in a picture and, above all, a proof that the system rested upon non-arbitrary foundations." (ibid., p. 11) The canvas was regarded as a window, often referred to today as Alberti window, through which the painter views the world and which intersects his visual cone (Lindberg, 1976). This gave rise to the idea that a realistic appearance of depth and space can be achieved in pictures by mimicking the exact geometrical relations in the structure of light that reaches the eye from a three-dimensional scene. Correspondingly, a system of construction rules gained prominence in Renaissance art as an artistic engineering technique for the purpose of creating on a flat canvas pictorial representations that induce a strong appearance of depth in the observer. Though these artistic techniques later joined with ideas on geometrical processes of image formation in the eye, their use and development were primarily shaped by considerations internal to the complex variety of cultural purposes underlying 
artistic productions. For the endeavour to imitate nature and to achieve visual truth in two-dimensional representations of the world the importance of rules for linear perspective is, however, on a par with those for simulating the effect of lights and the interaction of light and objects by using spatial pigment patterns on a flat surface (Schöne, 1954). It is a historically contingent development of art history that linear perspective rather than other aspects first gained prominence in this context.

With respect to linear perspective as well as other pictorial concepts like shadows or light, two different kinds of inquiries have to be distinguished. The one inquiry, pertaining to artistic craftmanship, concerns the physical generation process by which a three-dimensional scene can be mapped in a perceptually adequate and satisfactory way to a two-dimensional representational medium. The other inquiry, pertaining to perceptual psychology, concerns the way the visual system exploits the sensory input in terms of its primitives.

The techniques for generating pictures that imitate nature with respect to its spatial appearance do not touch upon, and actually had at their origin been thought of as independent of how the perceptual process, beyond its geometrical aspects, has to be conceived of. If we are interested in the principles underlying perception rather than in the simulation aspect, i.e. in the second kind of inquiry, we have to ask how the visual system exploits the Alberti window (understood as a physico-geometrical description of the sensory input), independently of how it was physically generated. This may lead to the heuristics to understand certain rules of linear perspective as belonging to the set of rules and principles internal to the visual system by which it exploits the sensory input in terms of its primitives. To derive, however, from the geometrical importance that rules of linear perspective have for relating a three-dimensional scene to its two-dimensional representation a distinguished status for these rules within perception theory would amount to succumbing to the physicalistic trap. I will shortly address two ways in which the physicalistic trap may be disguised, one pertaining to the socalled robustness of perspective, and one related to the notion of a perceptual space.

\section{The 'robustness' of perspective under changes of vantage point}

The term 'robustness of perspective' refers to the phenomenon that in viewing a picture a displacement of vantage point usually does not result in strong perceptual distortions, such as expansion, compression or shearing, of the 'virtual space', as would be expected by geometrical considerations on the bases of the original vantage point from which the picture was geometrically constructed. Like in other cases of so-called constancy phenomena, this way of formulating the problem is based on a pre-theoretical segregation of phenomena into 'normal' ones, and ones that are in need of particular explanation. The 'normal' case, viz. 
the perceptual appearance that is evoked by viewing the picture from the 'true' vantage point, is considered to be basically explained by referring to linear perspective. It is then noticed that this 'explanation' can not explain what happens when we view the picture from a point that considerably differs from the vantage point underlying its geometrical construction. Therefore the need arises to invoke additional mechanisms subserving robustness of perspective. This pattern of explanation is based on the presumption that the task of the visual system is to invert the geometrical process and to calculate backwards from the image the true three-dimensional layout of objects in a scene. With respect to the 'true' vantage point this does not constitute a particular problem and thus corresponding phenomena are considered to be not in need of a particular explanation. ${ }^{11}$ This is basically the inverse optics perspective (Poggio, 1990) according to which it is the task of the visual system to recover the structure of scene characteristics from characteristics of the sensory image by physico-geometrical computations. Ideas like this can be regarded as a complex variant of the measurement device misconception of perception. Though they can, in specific cases, provide interesting heuristics for the identification of internal rules, there is no evidence supporting the idea that internal spatial representations are based on inverting linear perspective. The 'true' vantage point is of no particular relevance for what has to be explained. Rather, what has to be achieved is an explanation that accounts for the structure of the percept elicited by a specific sensory input and its stability over certain variations of the input.

\section{The notion of phenomenal space}

The idea that the organism has to recover projective or metric aspects of physical space and derive an internal representation of this space that is adaptively transformed for its biological purposes is strongly rooted in a measurement device misconception of perception. There is a long tradition in the psychology of spatial perception - based on what Koenderink and van Doorn (1998, p. 297) describe as the "weird notion that we don't really see the world but only some deformed copy of it in our head" - to investigate the quantitative and metrical aspects of a presumedly homogeneous and uniform 'perceptual space'. ${ }^{12}$ This notion of a coherent visual space is so seductive that arguments pointing out that it is conceptually misguided and empirically inadequate (MacLeod \& Willen, 1995; Koenderink, 1998) have scarcely undermined its impact.

\footnotetext{
${ }^{11}$ For each image there is, however, an infinity of potential physical processes that causally may have given rise to it. These are, as Koenderink, von Doorn and Kappers (2000, p. 183) expressed it, "related by some group of 'ambiguity transformations'. What this implies is that the orbit of the fiducial scene under the group of ambiguity transformations is a 'metamer' of the fiducial scene. (..) Thus all metameric scenes have an equal claim on the epithet 'veridical'!" Whence they rightly state, "It is the 'veridicality' question that makes no sense." There is, thus, no notion of a 'true' vantage point that can figure in a formal theory of perception.
} 
Internal spatial representations have a rich but up to now poorly understood internal structure. They seem to be subserved by a rich structure of subsystems, pertaining to aspects such as occlusion, motion, shading, perspective, texture and stereo disparity. A sketch of a few lines, or patterns that give rise to the impression of one object partly occluding another or of an attached or cast shadow, patterns of texture etc., they all can elicit forms of internal spatial representations. Such representations seem to be part of the data format of perception, "the innate three-dimensional organization of visual appearances" (von Szily, 1921, p. 971). Though they are barely understood presently, the evidence available suggests that rather than being based on some fixed and homogeneous space in the sense of geometry these representations have a dynamic situation-dependent nature involving topological, projective and metrical aspects with a complex structure of parameters and interactions with other primitives.

\section{The dual character of pictures}

Ideas underlying the physicalistic trap in picture perception not only tend to generate pseudo-problems that result in a distorted theoretical picture of the nature of perceptual representations. They also increase the danger of concealing important structural properties of perception. An interesting case in point are phenomena that are discussed under the heading of the dual character of pictures. This term refers to the observation that pictures can generate an in-depth spatial representation of the scene depicted while at the same time appearing as flat twodimensional surfaces hanging on a wall (cf. Michotte, 1948). From a physicalistic perspective they refer to a kind of discrepancy between what is physically there, viz. a flat surface, and the perceptual impression evoked. Postulating such a discrepancy, however, rests on conflating the level of the physical generation process of the sensory input with the level referring to perceptual processes by which this sensory input is exploited.

\footnotetext{
${ }^{12}$ The idea of a 'personal phenomenal space', i.e. a joint coherent spatial representation underlying judgments of distance, movement or orientation, has been called The Tidy Mother model - 'a place for everything, and everything in its place' - by MacLeod. MacLeod and Willen (1995, p. 59) conclude from various empirical findings that "the notion of visual space, natural though it is, may not capture important realities of visual space perception."
} 
Rather than being a problem of a discrepancy between what is physically there and the percept, the observation that we can simultaneously handle in perception both the flatness aspect of the canvas and the spatial depth of the depicted scene seems to point to a pervading property of the cognitive system, which we can also encounter in various other domains, namely the ability of the system to handle what may be called conjoint representations over the same input (Mausfeld, 2002). Corresponding phenomena in picture perception show that two representations are not independent but interlocked, and that we can phenomenally accentuate one or the other aspect and switch back and forth in an effortless way (though such switches are correlated with depth aspects, they actually pertain to the entire perceptual organization of the visual field). The 'realities' of pictures as objects and depicted objects bear different amounts of internal computational relevance and phenomenological vividness. Similar observations can be made in many other domains, such as pretence play (a special case of acting as if, where the pretender correctly perceives the actual situation), in perspective taking (the ability to consider how objects, events or mental states are perceived from the point of view of another person and to simultaneously handle the egocentric and an allocentric perspective), or in language (where we can by some expression refer to something that is simultaneously abstract and concrete, cf. Chomsky, 2000). These cases appear to have interesting features in common and seem to point to an important property of highly complex perceptual and cognitive systems. Such systems have to subserve simultaneously a great variety of tasks and thus must internally have the outputs of many sub-modules available for purposes of stable higher-order representations, which naturally gives rise to conjoint representations over the same input. The internal handling of conjoint representations and their transformational structures can phenomenally be either imperceptible or it can be mirrored in multistability, perceptual vagueness, or a kind of book-keeping by double entry, as it were (see Mausfeld, 2003, for a more detailed account). 


\section{The physicalistic trap in functionalist and computational approaches to perception}

Within an elementaristic approach to perception, as exemplified by classical psychophysics, perceptual representations were investigated with respect to elementary physical variables that putatively had to be 'properly' perceived by the visual system. To the extent that it came to be recognized that the achievements of the perceptual system cannot be understood by using isolated elementary physical variables as a reference frame an important and influential conceptual shift in perception theory took place. The stage for these developments had been predominantly set by the Gestaltpsychologists, by Bühler, Brunswik, Michotte, and Gibson, and they culminated in the computational approach as pioneered by Marr. In functionalist and computational approaches perception theory has to start with an analysis and description of a specific perceptual achievement and then try to relate it, by a computational theory, to a suitable description of the available sensory input. Such computational approaches made it obvious that the perceptual system must have some primitives built in from the start (most investigations, however, exhibit a preference for a thin set of physically rather elementary primitives). Physical descriptions of the sensory input are then given in terms of these primitives and in terms of the biologically and perceptually relevant categories that are internally built up from these primitives.

In this respect, it is important to notice an essential difference between perception theory and machine vision: In machine vision the internal data structure and the physical properties and invariants, which are the basis of the system's achievements, are already determined in advance, i.e. machine vision deals with a categorically fixed and predetermined world. The contrary holds for perception theory: Its task is to identify what in machine vision is given by a fixed theoretical language, namely the internal data structure and the invariants in the sensory input that elicit the visual system's output, and thus to provide an explanatory theory of how perceptual concepts such as 'object', 'surface', 'event', 'illumination', 'transparency', 'shadows' etc. come about. Interestingly, it is precisely this problem that not only the elementaristic perspective, which is based on ideas from signal processing and communication engineering, has bracketed, but surprisingly also the functionalist-computational one. 
In order to succesfully deal with this problem one has to construct a conceptual framework for physically describing the relevant aspects of the world that is suitable for perceptual purposes, a framework that ties the physical description as closely as possible to the description of the perceptual mechanisms under scrutiny. This is what ecological physics is about. The development of the conceptual apparatus for describing the perceptually relevant physical aspects and of the one for describing the perceptual achievement have to go hand in hand, resembling the hermeneutic cycle in interpretative arts. Their 'natural kinds' can, of course, for principled reasons not coincide. However, a more suitable conceptual framework for ecological physics will greatly facilitate the development of successful explanatory theories of perception. It is still a major task of perceptual psychology to develop such a conceptual framework for describing perceptually relevant aspects of the physical world and thus of the sensory input.

Folk physics, though it is the necessary starting point for developing such a framework (cf. Smith \& Casati, 1994), is an inapt guide in this respect. Using physical categories from common sense taxonomies often results in the temptation to regard them as independent descriptions of the physical world that allegedly need to be 'recovered' from the sensory input in the process of perception. This, however, would amount to misconceiving the role of ecological physics and to bypassing the core problem of perception theory to identify the internal primitives and the categorial structure they give rise to. Nevertheless, corresponding ideas can be encountered in many current computational approaches that take the perceptual classification of the environment as pre-given and describe the external world by using concepts from folk physics, like objects, shapes, shadows, surfaces, or lights (mostly in refined forms as provided by ecological physics). However, these concepts themselves are formed by perceptual processes, and consequently cannot be viewed independently from them. Thus corresponding approaches are prone to surreptitiously using categories belonging to the yet-to-be explained perceptual categories for a description of the physical input while investigating how these categories can be 'recovered' from the sensory input. Hence, the question of what primitives are underlying those perceptual processes by which the physical world is parsed into perceptual categories, such as objects and events, is almost trivialized.

The case of apparent motion provides a simple example of the fact that perceptual and physical categories do not coincide. Furthermore, the existence of physical objects is not only not sufficient, but not even necessary for the corresponding percept (think of an object on a CRT screen or in a virtual reality setting). To lump together concepts of perceptual and physical categories and to 'explain' the former as a computational recovery of the latter is again an example of the physicalistic trap. Particularly the idea, already mentioned above, to conceive of "vision as inverse optics" (Poggio, 1990, p. 143) can be considered a legacy of the measurement device conception of perception (notwithstanding its merits for purposes of machine vision). 
The relations between properties of the external world that causally give rise to the physico-geometrical structure of the sensory input on the one hand, and between properties of the sensory input and internal outputs of the visual system on the other hand are, as mentioned above, two utterly separate problems that need to be distinguished and dealt with separately (Gibson emphasized the first part, Gestaltpsychology only dealt with the second part). The only physics of the external world that figures in a formal theory of visual perception is the physico-geometric properties of the incoming light array. Within such theories the level of analysis that vicariously represents the physical world is the Alberti window, understood as an abstract mathematical entity. An Alberti window is, intuitively speaking, a two-dimensional array orthogonal to a possible line of view of an observer. ${ }^{13}$ It is characterized by a spatio-temporal energy pattern caused by some physical processes. For reasons indicated before, notions like 'veridicality' do not figure in formal theories of perception. They only arise in (indispensable) ordinary or metatheoretical discourse when we, as researchers, make a guess about the most plausible physical causes of an Alberti window. Though formal theories of perception refer to properties of the mental structure of the observer only and have no place for notions such as 'reference to the external world' or 'veridicality', we cannot, in the development of such theories, dispense of heuristics derived from ecological physics.

\footnotetext{
${ }^{13}$ The concept of the Alberti window is a purely physical one and does not refer - as the concept of a proximal stimulus often does - to any properties of the observer. For the present purposes of conceptual clarification I can ignore problems that arise from the restriction to passive aspects of a merely visual input only, whereas in fact the internal data format is to a large extent transmodal and also reflects properties of an active observer.
} 


\section{Example IV: Bayesian approaches to perception}

There is a long tradition in perception theory, stretching from Alhazen through Helmholtz to Marr, that considers visual perception as essentially an inferencelike process by which certain conclusions about the external world can be drawn from the premises given by the information in the retinal image. In recent years important conceptual clarifications of these intuitions have been achieved and comprehensive mathematical frameworks have been developed (Bennett, Hoffman \& Prakash, 1989; Knill \& Richards, 1996; Kersten \& Schrater, this volume) that make these notions precise and allow their theoretical and empirical fruitfulness to be explored. The basic idea of these approaches can be described by reference to the Bayesian formula of inverting conditional probabilities: Vision is considered as being based on inferences by which scene properties are estimated from image properties. Since an estimation of scene properties is highly underdetermined by image properties, the goal of the visual system is, on this view, to derive the probability of various scene interpretations for a given sensory image and to base an appropriate decision on this information. The probability of a world scene given the image (posterior distribution) is basically given by the product of the probability of the image given the scene (likelihood function) and the apriori probability of the scene (prior distribution). The form of the prior distribution models prior knowledge that the visual system has available about the probability of different features of the world (which helps to substantially narrow down the degree of non-uniqueness of scenes that could have given rise to the image). A decision scheme that operates on the posterior distribution allows a unique interpretation of the image in terms of a scene to be derived. This decision scheme incorporates e.g. consequences of misclassifications, preferences for certain interpretations, or different accuracy requirements for different kinds of parameters. Using Bayesian decision theory for modelling inference processes in perception yields more general schemes than inverse optics (and corresponding regularization schemes) because a Bayesian framework can incorporate decision aspects as well as a greater variety of constraints.

Bayesian frameworks stress that the inference process must be couched in terms of 'world properties' (understood as perceptual concepts) rather than in terms of image properties ${ }^{14}$, and thus direct our attention to the problem of how to describe corresponding physical world properties in a theoretically fruitful way. They

\footnotetext{
${ }^{14}$ Bayesian approaches make clear that objects, 3D shape, texture, shading, motion or stereo depth are not the input but rather the output of the visual system. "In short, the table, and all properties of it that I experience, are my conclusions. What holds for tables also holds for forks, suns, brains, and neurons. These are the products of perception, not the antecedents. In perception, as a Bayesian would put it, we perceive only our posteriors." (Hoffman, 1996, p. 219/20)
} 
resemble the Gibsonian approach - rather than traditional psychophysics - with respect to the chosen level of physical description in that they tie the physical description to the macroscopic scale of environmentally and perceptually relevant categories. They, thus, do not refer to those concepts that have proved explanatorily useful for physical theory as such, but rather to a kind of relational or ecological physics that deals with physical descriptions of the categories that an organism employs in perceptually segmenting its environment.

Bayesian approaches, however, do not explicitly address and are neutral with respect to a core problem that we are faced with in the context of perception theory, namely the question of which conceptual vocabulary to use to describe in a theoretically fruitful way relevant properties of the image and relevant properties of the world. Shall we describe the image in terms of pixels, or geometrical entities, or natural objects, and thus base the inference process on luminous flux, on edges, or on meaningful objects, respectively? The vocabulary we use for the description of the input has to be as close as possible to the 'semantic' distinctions that the visual system makes, because computational operations on the incoming light array cannot result in a unique set of new meaningful perceptual categories, unless these categories are already built-in as primitives. ${ }^{15}$ What holds for the description of the image also holds for the description of the scene or the external world. In what terms can we formulate an explicit model of world structure? Apparently innocent locutions such as "The world we live in is a very structured place" veil the problem that what we have to refer to in models of world structure are not propositions about the physical world as such but about the world as structured by the yet-to-be-explained perceptual system of an observer. In which way, then, can we, without trivializing the problem of perceptual structure, make use of categories that describe the output of the visual system for a physical description of the input? Only if we assume that the categories for linking image events to world events, and thus the categorical structure of the priors itself, are given from the start and are built-in into the system. ${ }^{16}$ It is the structure of the internal representations that relates image structure to world structure (a relation that can be contingent from the point of physics or geometry).

This problem of chosing an appropriate set of internal primitives also extends to the specification of priors. The priors not only capture statistical dependencies between physical properties of the environment but also crucially refer to the

\footnotetext{
${ }^{15}$ This is essentially Fodor's (1975, p. 80f.) argument that one cannot, by some mechanism of inductive inference, acquire "a conceptual system richer than the conceptual system that one starts with", i.e. a conceptual structure "whose predicates express extensions not expressible by predicates of the representational system" whose employment mediates the acquisition.

${ }^{16}$ Even an extreme version of empiricism would have to assume that there is a set of given representational primitives; it would, however, assume that this set is exhausted by concepts used for the physical description of the sensory input.
} 
conceptual perceptual structure of the observer. The primitives that define the data format of internal processing and the structure of internal representations will dictate a core set of priors.

Current Bayesian approaches, instead of making this problem explicit, refer to the indefinite commendation to use 'higher level descriptions' of image and scene. They focus on the probabilistic inference structure linking image properties and world properties while sidestepping the problem of identifying the concepts and primitives that these processes operate upon. The general approach is succinctly described by Hoffman (1996, p. 220): "What we in fact do is to fabricate those priors (and likelihoods) which best square with our posteriors." Though this is necessary in order to make the Bayesian apparatus work, it conceals a core problem of perception theory that has to be tackled on its own. Richards (1996, p. 228) legitimately deplores that aspects of "design and the creation of meaningful cognitive structures" receive little discussion in these developments. Rather, current Bayesian approaches sidestep such aspects by trading upon an equivocation of terms such as surface, object, event surface colour, illumination colour, etc. which denote both physical categories for describing the input as well as perceptual categories for describing the output of the visual system (as revealed by using the same variable 'scene' in the posteriors and in the likelihood function). Because of this, current Bayesian approaches are strongly imbued with ideas from the inverse optics perspective and measurement device misconceptions, as is revealingly illustrated in typical phrases such as "the visual system attempts to estimate properties of the scene". ${ }^{17}$

However, once we have achieved a better understanding, beyond physical common sense taxonomies, of the internal categorial structure that gives rise to the posteriors, the general framework offered by Bayesian approaches has the attractive feature of jointly dealing within a common mathematical framework both with the internal structure of perceptual representations and with descriptions by ecological physics of relevant aspects of the external world. The Bayesian framework provides an inductive logic for describing the way in which the exploitation of given perceptual capacities and a given conceptual structure of perception varies with variations of the sensory input. It cannot, however, teach us anything about what the conceptual structure of perception is nor, in particular, derive it from physical descriptions of the sensory input.

\footnotetext{
${ }^{17}$ The Bayesian framework as such, however, does not suggest these interpretations and is, in fact, perfectly compatible with sign theories of visual perception. In particular, an internalist version of the Bayesian framework can be formulated, which specifies computational procedures that mediate the relation between the sensory system (which deals with the transduction of physical energy into neural codes and their subsequent transformations into codes that are 'readable' by and fulfil the needs of the perceptual system) and the perceptual system (which contains, as part of our biological endowment, the rich perceptual vocabulary - which extends far beyond physical aspects of the external world - in terms of which we perceive the 'external world').
} 


\section{Example V: Colour constancy from a computational point of view}

According to elementaristic approaches to colour perception the alleged goal of the visual system is "to estimate the spectral shape of the incoming colour stimulus". Elementaristic approaches tie colour representations to the physical nature of the sensory input. In order to account for phenomena of approximate colour constancy of objects under varying illumination secondary 'compensation mechanisms' have to be invoked. This way of carving the problem of colour perception had already been criticized by Hering (1920, p.13): "Vision is not a matter of perceiving light rays as such, but the ability to see external objects by means of these rays; the eye's task is not to inform us about the respective intensity or quality of the light that comes from the external objects, but to inform us about the objects themselves." Bühler, Heider, Brunswik, Kardos and others have since then pursued similar functionalist perspectives on perception, which culminated when they merged with a computational approach, as pioneered by Marr. A functionalist-computational approach regards it as crucial for attempts to understand the structure of internal representations to start with an appropriate conjecture about the purpose for which they are used. Such ideas together with corresponding distinctions of levels of analysis constitute an important conceptual step in the development of the field. Since we are, however, far away from understanding the purposes of the various components of the visual system, we encounter immense problems when we try to substantiate these ideas in concrete cases. In such situations it is seductive to derive, by mis-idealizing the perceptual achievement, purported goals from physical considerations. This is what had happened with respect to colour perception, as exemplified by the assertion that basically "the goal of colour vision is to recover the invariant spectral reflectance of objects (surfaces)." (Poggio, 1990, p. 147) The idea that the structure of internal colour representations is determined by the computational goal of recovering from the sensory input a function that depends only on certain physical properties of objects, viz. characteristics of surface reflectance, though, of course, not without heuristic value, is patently a distal variant of the measurement device misconception of perception. Corresponding approaches are encumbered with both empirical and theoretical problems.

Concerning their empirical adequacy they impute to the visual system a goal that is not consonant with its actual achievements. What is achieved is not an estimation of spectral reflectance functions, but rather an abstractive categorial description of the 'colour of a perceived object', which is more stable than can be expected on the basis of the local sensory input, i.e. the wavelength composition of the light coming from the object to the eye. In this sense, the percept 'colour of an object' seems to be more strongly tied to the spectral reflectance characteristics of the object than to the wavelength composition of the local sensory input. There is, however, no colour constancy in the strict sense that two locations of the same spectral reflectance 'look the same' under two different illuminations. One can see 
the 'same colour' but yet have a different colour experience by seeing it under a different illumination. The phenomena concerning the interplay of surfaces and illumination in colour perception, e.g. Helmholtz' observation that "colours that can be seen at the same location of the visual field one behind the other", point to much deeper principles of the visual system than those of some re-normalizing of the local colour code or the visual system's alleged propensity to keep its colour equivalence classes congruent with the physical structure of 'reflectances of surfaces' (cf. Mausfeld, 1998). In the early literature several promising attempts were made to identify these structural principles, e.g. by Bühler (1922), Gelb (1929), Koffka (1932), MacLeod (1932) or Kardos (1934), attempts that have almost been completely forgotten under the influence of the sensory data processing approaches that since then have become the dominant paradigm in colour perception, be they neurophysiologically oriented in the sense of neural data processing or computationally oriented in the sense of sensory image processing guided by some functional goal. Corresponding theoretical distortions have been facilitated by our lack of a suitable theoretical language for the phenomenal description of the percepts associated with the interplay of perceived illumination and perceived objects, since such a description has to deal with aspects of, for instance, vagueness, abstraction and categorization.

From a theoretical point of view the computational approaches to colour perception mentioned throw together two different levels of analysis. It is one question to ask what properties of the environment give rise to perceptually relevant properties of the Alberti window (or to study what, from an evolutionary point of view, is the 'normal explanation' for the structure of the Alberti window), and a completely different problem to investigate how structural properties of Alberti windows are exploited by the visual system in terms of its primitives.

The same characteristics of a light array reaching the eye can be physically produced in many different ways. With respect to the percept 'surface under chromatic illumination' the same spatio-temporal light pattern that is caused by a certain interaction of physical surfaces and light sources and that elicits corresponding percepts can be produced by light sources alone (using, for example, a slide or a CRT screen). The visual system cannot distinguish these cases, it simply doesn't know whether the causal chain giving rise to this pattern arises from surfaces and light, or lights alone. A goal of perceptual psychology is to identify the equivalence classes of input patterns that give rise to the same internal outputs and thus to provide an abstract explanatory framework for the structure of perceptual representations. A description of such equivalence classes in the language of physics will very likely lead to very abstract mathematical entities that are quite unnatural from the point of both theoretical physics and folk physics. This again highlights the futility of attempting to provide a description of the equivalence classes of colour codes in terms of their possible physical causes; colours do not constitute a well-formed physical kind. The internal structure of colour perception is given by the structure of representational primitives in which parameters for 'colour' figure. These primitives determine the structure of internal 
colour codes, and thus the kind and structure of equivalence classes of input codes that result in corresponding output codes. Because the equivalence classes are 'held together' by the structure of our perceptual system, rather than by the structure of the physical environment itself, any reference to the potential distal causes of the Alberti window is extrinsic to a formal theory of colour perception. Again, notions of representational content or reference to the environment do not figure in formal theories that provide explanatory frameworks for our understanding of the internal structure of colour. The question of whether colours 'represent' what they normally stem from in our environment is of no relevance for our formal theories of perception, though corresponding considerations are, of course, an indispensable part of our metatheoretical talk about colours.

The structure of internal representations cannot be revealed by referring to physical properties like surface reflectance characteristics from the outset because there are no such things in the Alberti window. They cannot even be assumed to be necessary causes for the corresponding categories. Internal concepts, such as 'surface colours', are not constituted by the corresponding categories of physics or tied to them e.g. in the sense of the latter being necessary and sufficient conditions for the former. Rather they are constituted by biologically relevant features of the environment as well as by internal constraints and requirements of the entire perceptual architecture. Not much is known today about the 'internal semantics' (as a purely syntactically-defined feature) of the visual system, but there are good reasons to assume that basic 'semantic' units of perception are predetermined and tied to certain spatio-temporal characteristics of the incoming energy.

Experimental and theoretical evidence strongly suggests, however, that the segregation into 'surface colour' and 'illumination colour' is not something that is derived from an analysis of the 'physical scene' but rather a primordial format of organization of representational primitives in which 'colour' figures as parameter. The perceptual system exploits the incoming light array in terms of its representational primitives. For instance, what can be described as an 'inference' whether a chromatic deviation of the space-averaged colour codes from some neutral point is due to a 'non-normal', i.e. chromatic, illumination or due to an imbalanced spectral reflectance composition of the scene has to be achieved by a specific activation of representational primitives by signs that the sensory system provides on the basis of relevant reliable regularities of the incoming light array. We found evidence that second-order statistics of chromatic codes of the incoming light array differentially modulate, by a specific class of parametrized transformations, the relation of the two kinds of representational primitives involved (Mausfeld \& Andres, 2002). 


\section{Example VI: Evolutionary internalized regularities of the physical world}

There are many other cases in which the physicalistic trap imbues the way problems of perception theory are formulated. As a final example I will briefly address an approach of current interest that comes under the heading 'evolutionary internalized regularities' (see also Bennett \& Hoffman, this volume) and has been prominently advanced by Shepard (e.g. 1987, 1994). This approach connects inquiries into the internal structure of perceptual representations with conjectures about physical regularities that may have phylogenetically become mirrored in perceptual structure. Such regularities may refer to "the fact that space is three-dimensional, that objects have six degrees of freedom of global motion, that light and dark alternate with a fixed period, and that sets of objects having the same significant consequences tend to form a compact region in an appropriate parameter space." (Shepard, 1987, p. 269) We can distinguish various types of relevant regularities, such as general physical regularities (like gravitation or the three-dimensionality of local space), physico-geometrical regularities resulting from a specific relation between observer and environment (like perspective geometry), physically contingent global regularities (like the spectral composition of sunlight), and local niche-specific regularities. The urge to look for internalized physical regularities arose from the observation that there is a huge discrepancy between the sensory input and the perceptual achievement and that the structure of perceptual representations cannot simply be accounted for by the information available in the sensory input. It is thus occasioned by the same fundamental insight that resulted in the invocation of concepts like 'unconscious inference', 'higher order' processes, Gestalt principles, or 'internalized knowledge' 18 , an insight that time after time has been obstructed and disowned by empiristic ideology. One can hardly overestimate the strength and longevity of the behavioristic and empiristic tradition. ${ }^{19}$ Its core element, viz. the disregard for mental structure, still provides, in various modern disguises, the basis for much of current thinking about perception. Hence, referring to mental structure and concepts in perception theories is still considered by many as anathema. In contrast, Shepard clearly recognized the explanatoric vacuum caused by the prevailing disregard for the internal structure of mental representations and emphasizes the need for exploring the properties of the rich internal structure of the perceptual system. Taking serious the idea that we need to postulate, within explanatory theories of perception, a rich internal structure which the perceptual system is endowed with will have radical consequences for the kind of questions

\footnotetext{
${ }^{18}$ Rock (1983, p. 326)

${ }^{19}$ The empiristic stance that pervades current inquiries into perception and other kinds of mental activity and which emphasizes plasticity and variations due to individual and cultural learning history seems to be a natural element of our common sense reasoning about mental activity that we illegitimately transfer to scientific inquiry (cf. Chomsky, 2000, p. 163).
} 
asked in perception theory. Shepard, who has reinvigorated psychological inquiries into the structural form of mental representations, seems to eschew these radical consequences and resorts to the idea that the rich structure is imprinted on the mind of the perceiver almost entirely from without by the evolutionary internalization of external physical regularities.

As a research strategy heuristics pertaining to evolutionary internalized physical regularities have, of course, much to offer. They exhibit again the explanatory gap between the meagre sensory input and the exceedingly rich perceptual output, and generate theoretical speculations that can enrich, in concrete cases, our knowledge about the internal structure of perception. Corresponding endeavours bear a close resemblance to and extend inquiries into ecological physics.

Notwithstanding the potential fruitfulness of such heuristics, we have, with respect to perception theory, to distinguish the level of analysis that pertains to the individual organisms in a specific context (or to a submechanism subserving a specific function) from the one that pertains to the corresponding evolutionary history. The first level of analysis aims at an explanatory account of the structure of perceptual representations for a given organism. The second level pertains to the question, whether the structure of internal representations can be related to specific physical regularities of the environment that have been internally encoded in evolutionary history and are used for or constrain internal computations. ${ }^{20}$ In an successful explanatory theory of perception, based on an appropriately rich set of internal primitives, no need arises to refer to some internalization of physical regularities. Here, as elsewhere in biology, a satisfactory ahistorical account for a functional structure does not ipso facto suffer from some kind of explanatory deficit. Furthermore, evolutionary speculations about cognitive structure are encumbered with well-known problems (e.g. Lewontin, 1990); presently we know next to nothing about the specific evolutionary mechanisms and about the physical or functional constraints that are responsible for the development of the internal structure of perception nor about the features, the selection for or of which has shaped the structure of internal representations. Obviously, the organism as an entirety has been adapted to the specific circumstances and properties of the environment in which it has evolved; from this it does not follow, however, that the structure and the properties of specific perceptual subsystems are primarily determined by and 'optimally' adapted to specific features of the environment.

Regarding evolutionary aspects as external to an explanatory account of the nature of perception and as belonging to metatheoretical discourse, does not, of course, amount to denying any dependencies. The question is not, how in reality things are related to each other; perception is related to and dependent on various aspects of reality like phylogenetic development, metabolism, the immune system

\footnotetext{
${ }^{20} \mathrm{cf}$. footnote 13
} 
or the physics of the brain. The question rather is what constitutes an appropriate level of idealization for successful explanatory frameworks of perception.

Furthermore, it is not clear what is meant by the notions of 'physical regularity' and 'internalization'. Shepard (1987, p. 269) has been arguing "that to the extent that the principles of the mind are not merely arbitrary, their most likely ultimate sources are the abiding regularities of the world." Of course, understood in the wider sense that principles of the mind have evolved within the regularities of the world and are ultimately themselves part of the regularities of the world, this assertion is true, but of no theoretical interest. In a more specific sense it can be understood as the assertion that the structure of internal representations is determined first of all by regularities of the external physical world, whereas no essential explanatory importance is attached to factors such as behavioral regularities of conspecifics, to internal physical and architectural constraints or to idiosyncratic properties of the cognitive architecture. It is indubitable that we can, in many cases, find some external regularities to which properties of internal coding 'conform' but nothing about internalization is implied by that. But it is Shepard's main point that regularities of internal representations are due to an internalization of external physical regularities, and that the most abiding regularities under which the system has evolved are good candidates for regularities that have become internalized. Even if we would accept, despite the lack of corresponding empirical evidence, the assertion that, in general, principles of internal representations are determined by external regularities, we are faced with an intrinsic indeterminateness of the set of potential "candidates for regularities that have become internalized." We can formulate literally infinitely many physical regularities, i.e. relations on sets of physico-mathematical entities that remain invariant under certain sets of transformations, of any degree of 'unnaturalness', under which the perceptual system has evolved. What the intuition behind the notion of regularities seems to be aiming at, however, is something like 'natural' physical regularities that can be related to but are, in principle, independent of the specific perceptual design of the observer. These are understood as providing constraints that have been phylogenetically internalized by the perceptual system and determine the structure of its internal representation. There is, however, no apriori notion of organism-relevant physical regularities. What is a regularity depends on the structure of the organism under scrutiny, such as its size, the spatial and temporal integration properties of receptors and other neural structures, the properties of its memory and its representational capacities. The structure of the organism determines which regions of the parameter space of the physical world are regarded as an environment. Concepts such as 'regularities' or Barlow's 'redundancies' and 'suspicious coincidences' can, in the context of perception theory, only be defined relative to given representational capacities of the respective organism. Corresponding considerations inevitably lead back to the core problem of perception theory, viz. to understand the internal conceptual structure of 
perception, a problem that cannot be dodged by referring to physico-geometrical or statistical regularities of the physical world.

It is noteworthy that Shepard's primary motivation for postulating an internalization of features of the physical world is not to enable the system to solve an otherwise highly underdetermined task. His major thrust rather is to provide an explanation for the specific structural form of internal representations. In doing so he refers to principles that "reflect quite abstract features of the world, based as much (or possibly more) in geometry, probability, and group theory as in specific, physical facts about concrete material objects" (Shepard, 1994, p. 26), and ties the internalization process to the evolutionary advantage that "genes that have internalized these pervasive and enduring facts about the world" (ibid., p. 2) ultimately have. His approach thus extends the approach of ecological physics to more abstract mathematical descriptions of regularities of the world, which can be used then as heuristics for exploring the structure of internal representations. Still, the critical ambiguity about the internal role of external regularities that can be found in standard functionalist-computational approaches translates into his approach. The perceptual system can take advantage of a physical regularity in the sense that the way internal mechanisms work is based on the existence of specific physical regularities. In this sense we can say that the regularity is mirrored in the design of the system, as in the case of "the hawk and the ground squirrel (who) have internalized the period of the terrestrial circadian cycle." (ibid., p. 2) Being based on or taking in evolutionary history advantage of a physical regularity does not imply, however, that the system has internalized the regularity in the sense that it is explicitly encoded or used in some internal computations.

Approaches based on the notion of internalized physical regularities rightly acknowledge that we have to assume a rich internal structure of the perceptual system in order to account for its output. They thus draw our attention to a core problem of perception theory, viz. to understand the structural form of internal representations. They claim, however, that the structure required can be borrowed from, possibly highly abstract, external regularities. The danger, again, is that one uses physical regularities of the external world not just as an important heuristics about the structure of internal representations but rather projects them, as it were, into the perceptual system as a surrogate for internal structure. 


\section{Perception theory beyond the physicalistic trap}

Still, the only reasonable way, it seems, to embark on investigations about perceptual representations, is to venture ideas about the aspects of the physical world that are internally represented by the perceptual system. After all, the perceptual system subserves the function of coupling the organism in an adaptively appropriate way to the external world. Therefore, internal perceptual representations cannot be 'inconsistent' with biologically important physical regularities of the world. Thinking along the lines of physical descriptions of the external world then is, one might argue, the only means to understand the nature of perceptual representations, rather than being a trap. How else could we, according to this claim, arrive at a theoretical understanding of perception than by trying to identify the physical regularities to which the system is tied and which are internally mirrored in its machinery? In a very general sense, this is, of course, true; it simply rephrases, however, the general definition of perception. Also, it is indisputable that considerations both from phenomenology and from folk physics, including ideas about veridicality, are an indispensable part of our metatheoretical discourse about perception. The trouble arises when we try to derive more specific formulations from this idea. Even if we simply restrict our attention to the physical side of perception, i.e. to perceptually appropriate physical descriptions of the external world, the general idea that the function of perception is a coupling to the external world does not provide a clue to which set of physical concepts, from infinitely many, we use for a description of the external world and of physical regularities. Should we couch a description of biologically relevant aspects of the physical world in terms of e.g. quanta, light rays, spectral energy distributions, optical flow, surfaces, or physical descriptions of semantic categories, like 'edible things'? Any (conjectural) decision has to be based on arguments provided by perception theory. Neither descriptions based on elementary physical variables nor those based on common sense classifications as underlying folk physics are exempted from such a justification. The physicalistic trap amounts to assigning such descriptions a higher degree of plausibility and using them in a somewhat apriori way not only for describing allegedly relevant physical aspects but also for theoretically segmenting perceptual structure.

However, any successful explanatory account of perception has to be based on a physical description of the external world that is tied as closely as possible to the (yet-to-be identified) conceptual structure of the perceptual system.

A problem of even greater concern for perception theory is that the structure of perceptual representations cannot be understood if we restrict our attention to the physical side of perception, however appropriately we have chosen our vocabulary for describing the external physical world. The structure of internal representations is shaped not only by regularities of the external physical world, 
but also by biological regularities (e.g. the behaviour of conspecifics) that are contingent with respect to physics, by internal physical and architectural constraints, and by contingent properties of internal coding. As mentioned in the beginning, it is not sufficient for perceptual representations to be adequate with respect to the external world, they must also be functionally adequate, in the sense that they have to fit into the entire perceptual architecture including its interfaces with the sensory system, the motor system and the higher cognitive system, where meanings are assigned in terms of 'external world' properties. It is a characteristic feature of the physicalistic trap that it ignores these aspects and overlooks to what extent perceptual representations are structured by internal constraints. Presently we know much less about these other constraints than we know about aspects of ecological physics. ${ }^{21}$

Many early writers, notably the Gestaltists, Michotte, and ethologists, were aware of this and rightly emphasized the rich internal structure of perceptual representations by which the organism imposes a structure on the sensory input that cannot be derived from an analysis of the physical input alone. They did, however, not have the rich conceptual tools made available today by ecological physics and computational approaches to put these insights to work. Due to the prevailing empiristic presuppostions most of these insights have been forgotten or even ostracized. Therefore, approaches that tend to regard the perception process as being based on the information, with regard to external environmental contraints, available in the sensory input (plus some 'generalized learning mechanisms') still dominate the field. These approaches typically share an extremely empiricist point of view with respect to mental structure.

In contrast, ethologists, such as v. Uexküll, Lorenz and Tinbergen, have marshalled - taking the entire organism as the level of analysis - an impressive array of observations in support of innate and phylogenetically shaped building blocks of behaviour. With respect to perception we can find approaches similar to or inspired by ethology in perceptual psychology (e.g. Heider, 1930; Tolman \& Brunswik, 1935), in neuroethology (e.g. Barlow, 1961) and in computational neuroethology (e.g. Hassenstein \& Reichardt, 1956; Gallistel, 1998). Ethological investigations have suggested a theoretical picture according to which perception cannot be understood as the 'recovery' of physical world structure from sensory structure by input-based computational processes. Rather, the sensory input serves a dual function: firstly, it provides triggering cues for which representational primitives are to be activated, and thus selects among potential data formats in terms of which input properties are to be exploited. Secondly, it triggers processes that result in a specification of the values of the free parameters of the activated representational primitive. (Thus, even 'highly impoverished' sensory inputs can trigger perceptual representations whose 'complexity' far

\footnotetext{
${ }^{21}$ An intriguing example of an internal functional constraint is the principle of genericity, which favours non-accidental interpretations over accidental ones (cf. Albert \& Hoffman, 1995).
} 
exceeds that of the triggering stimulus and whose relation to the sensory input can be contingent from the point of physics or geometry).

The tension between intuitions that regard visual perception as essentially being based on inverse optics-like computations and those that regard it as being based on a rich structure of given primitives and internal computations that are triggered by the sensory input can be traced back to the beginnings of perception theory (see Appendix). It is important to note that intuitions about a triggering of perceptual representations must not be understood as being based on the idea that the mind is a repository of prefigured and innate ideas and that the sensory stimulus elicits something from this internal storehouse of ideas. Rather they refer to the observation that while the sensory input is a causally necessary requirement for perceptual representations, the perceptual computations triggered are under the control of an internal programme based on a set of representational primitives. ${ }^{22}$ Perceptual computations are thus representation-driven rather than stimulus-driven. They mirror the way perceptual representations as an entirety are organized, rather than being locally tied to the physical variables that causally gave rise to the sensory input.

There is hardly any disagreement among theoretical perspectives on perception that our visual system must be based on some representational primitives that are built-in from the start; however, different theoretical approaches strongly disagree about the question, how rich and complex - in relation to physical descriptions of the sensory input - these primitives have to be assumed to be. Though we are far from having a clear theoretical picture about the structure of primitives underlying perceptual representations, available theoretical and empirical evidence strongly suggests that primitives such as 'surface', 'object', or 'event' are among the pillars on which the structure of perceptual representations rests. ${ }^{23}$

\footnotetext{
${ }^{22}$ A felicitous description is, with respect to the mental entity 'pictorial relief', given by Koenderink et al. (2000, p. 184); "The picture acts as a 'constraint' on the beholder's creativity." Koenderink et al. speak of a "true creative force and not simply a bag of tricks. (..) It is much as with 'Gestalt laws' of 'early vision': They are spontaneously acting (creative) forces rather than simple 'filters'." Three hundred years earlier Cudworth (see Appendix) expressed basically the same thought: "Sense is but the offering and presenting of some object to the mind, to give it an occasion to exercise its own inward activity upon." In perceptual psychology similar ideas have been express time after time; they have suggested themselves first and foremost by the observation of the "wide gulf between sensory stimulus and percept" (von Szily, 1921, p. 971). The task of perceptual psychology then is to develop an explanatory framework to account for the "creative forces" (ibid., p. 971) of the perceptual system that mediate between sensory input and percept.

${ }^{23}$ Among representational primitives pertaining to 'objects' are, as corresponding evidence suggests, not only those that pertain to 'physical objects' of various types but also a great variety of specific types that pertain to categories such as 'intentional physical objects', 'potential actors', 'self', or 'other person'. Representational primitives pertaining to 'events' can be expected to exhibit, as temporal analogs to 'objects', a corresponding variety of different types.
} 
A wealth of observations, stretching from Gestaltists' observations on figureground segmentation, to Gibson's ideas, to computational approaches indicate that among the representational primitives of the perceptual system 'surface' plays a distinguished role (see Nakayama, He \& Shimojo, 1995, for a more recent account). Perceptual representations are organized in terms of 'surfaces' among which certain ones can play special roles, notably the ground plane (Sedgwick, 1986). As representational primitives, 'surfaces' have a variety of perceptual attributes, such as depth, orientation, colour, brightness, texture, etc. (understood as internal concepts), that have to be specified by the incoming sensory information. The formats of these attributes are determined by the internal architecture; they are, in line with Müller's law of specific nerve energies, given by design. Their concrete values, however, are dependent on the specific sensory input and on the perceptual representations to which they are attached. We can conceive of them as free parameters attached to certain primitives and as part of the representational format of these primitives. For example, in the case of colour we have, accordingly, to distinguish different types of colour parameters, depending on the particular primitive to which they belong. 'Colours' that are attached to an internal representation of the transmission medium subserve a different function and exhibit different coding properties than 'colours' attached to the internal concept 'surface'. Which parameters are specified in which way and which associated classes of transformations are activated (pertaining e.g. to the idealized functional goals of 'scene invariance' or 'illumination invariance') is then determined by specific physico-geometrical properties of the sensory input (Mausfeld \& Andres, 2002; Mausfeld, 2003).

On the basis of currently available evidence successful explanatory accounts of perception not only have to be based on postulating a sufficiently rich set of structural primitives such as 'object' or 'event' of various types - which specify the internal data format, as it were - but also on domain-specific relational and transformational primitives pertaining to e.g. similarity, identity, continuity, coherence, to a variety of spatial and temporal relations, to topological properties, or to the requirement of guaranteeing smooth transitions between internal representations. Prominent examples of relational primitives are 'causality' in the case of physical entities (Michotte, 1946; Scholl \& Tremoulet, 2000), and 'intention' in the case of mental entitites (Premack \& Premack, 1995).

While the theoretical picture of the basic principles underlying perception that is emerging is still very skeletal and of necessity has to be based on considerable theoretical speculation, it receives support also from more recent study of the newborn and young children in developmental psychology. Corresponding studies provide convincing evidence that our mental apparatus is, as part of our biological endowment, equipped with a rich internal structure pertaining to e.g. structural knowledge about properties of the physical world, to distinguishing 
between physical and biological objects, or to imputing mental states to oneself and to others. ${ }^{24}$

Our perceptual apparatus serves to couple the organism to biologically relevant aspects of the external world. For an organism with a mental structure as rich as ours the relevant aspects of the 'external world' do not only pertain to physical and biological aspects but also to the mental states of others. We perceive not only colour, shape, depth, and physical relations as causality, but also emotional states and intentions of others. With respect to the architecture and functioning of the perceptual system there is no fundamental difference between perceiving physical aspects of the external world or aspects of the mental states of others. In both cases the sensory input serves as a sign for biologically relevant aspects of the external world that elicits internal representations on the basis of given representational primitives. Though we are still far from understanding the structure of perceptual representations and their role within mental architecture, there are good reasons to assume that perceiving physical aspects like shape, colour or depth is not more direct or immediate than perceiving mental states of others. Once we recognize that both rely on the selfsame basic principles we are able to ask novel and promising questions about the internal representational structure of perception.

\footnotetext{
${ }^{24}$ From the extensive set of investigations that are of relevance in the present context I will only mention Landau, Spelke \& Gleitman (1984), Carey \& Spelke (1994), Spelke (1995), Meltzoff (1995), and Trevarthen (1998).
} 


\section{Appendix}

\section{Historical annotations on the notions of sensory data-based computation vs. triggering in perception theory}

The tension between intuitions about sensory image-based computation-like processes in perception (i.e. transformations based on the same theoretical vocabulary) and triggering theories of perception (i.e. transformations based on different sets of primitives) goes back to the earliest conceptual developments in the history of perception. ${ }^{25}$

According to ancient conceptions of perception things imprinted themselves (influxus physicus) as entire objects into the soul by some forms (species sensibiles) which emanated from physical objects. Thus, no distinction between sensation and perception could have been made. However, similars can only be perceived by similars (similia similibus percipiuntur). Therefore some tertium quid must exist like in eidola conceptions - that mediates between the dissimilar instances of soul and material objects. Building on these ideas, Alhazen and Kepler assumed that the senses transmit images of objects to the mind and that consequently perception is the mental correlate of the retinal image.

Alhazen was aware, however, that the process of visual perception cannot solely be understood on the basis of the geometrical processes involved. Rather, he assumed that non-geometrical mental operations have to be involved. It is interesting to note that the assumption of inference-like mental operations opens the way for an entirely new conception of perception, namely perception as mediated by signs. Alhazen noted that properties of objects like shape, position, size or movement can be perceived "only by inference and signs" (Optics, Bk. I, $6,60)$. For Alhazen a sign is, as Sabra (1989, p. 80) put it, "a distinctive mark or feature or property of an object which serves as an index or clue of the object's identity." Perception by signs is possible because "the forms of all familiar objects and species, and of all common properties, have been established in the soul and shaped in the imagination and are present in memory." Perception of such properties therefore can be achieved "by means of signs and prior knowledge" (Optics, Bk. II, 4,22). It is important to note that visual inferences based on signs can refer to cues that are not supplied by the optical images themselves. Though Alhazen's idea of inferences based on signs differs from the much more radical (but very different) conceptions of a sign or symbol theory of perception proposed

\footnotetext{
${ }^{25}$ See Atherton's (this volume) historical analysis of the distinction between vision based on natural geometry and calculation-like processes vs. perception by signs, and Hatfield (this volume) for a critical discussion of approaches that attribute sophisticated physical concepts to the visual system.
} 
later by, say, Cudworth, Reid or Helmholtz, his distinction between two kinds of inference, one based on signs (comprehensio per signum; Optics, Bk. II., Ch. 3, 24,25, Ch. 4, 20-25), the other on a thorough inference performed by the "faculty of judgement" (per aliquam modorum ratiocinationis, Optics, Bk. II, Ch.3, 20) was an important conceptual step that paved the way for later developments.

A corresponding distinction of two such different processes can also be gathered from Descartes' writings. For Descartes the material object does not cause a physiological copy which then serves as the immediate object of perception. Rather the mechanistic physiological process is only a means of perception that arouses certain ideas in the mind. Thus, the movements of the nerves only act as signs that - mediated by movements of the pineal gland - stimulate certain mental ideas (which corresponds to Descartes' second grade of sensation). (As Atherton, this volume, states, "part of his project is to argue that the retinal image qua image plays no role in sensation.") These movements do not resemble what they represent. As a kind of blending of bodily and mental states (confusi quidam cogitandi modi, Meditationes VI, 13), the sensations which were aroused in the pineal gland do not resemble external objects, in the same way that words do not resemble the objects that they denote. This has the important consequence that not even sensory concepts can be derived from experience. ${ }^{26}$ Also form or size are abstract ideas that cannot directly be derived from the senses. Rather some 'raw ideas' of size, form etc. are triggered by certain excitations of the senses. The structural relationship between these ideas is assumed to resemble the structural relationship between the corresponding external qualities. In this sense, Descartes seems to assume, that the 'real' properties of the external world are completely divorced from the corresponding ideas in the mind. On the other hand, however, he holds that there are processes (which do not operate on the retinal image, but within the domain of abstract ideas) that allow us to achieve (partly and imperfectly) access to properties of external objects. In the wake of Alhazen, Descartes postulated calculation-like processes based on natural geometry, by which we can achieve judgements of a higher degree of reliability of the corresponding properties of the external objects. (cf. Hatfield's account, this volume, of the corresponding ideas of Alhazen and Descartes.) The caginess and even incoherence of Descartes' corresponding writings indicate that Descartes was loath to varnish over the huge complexities that he identified in his attempts to provide a new explanatory framework for the relationship of the mental and the physical. Malebranche was more willing to sacrifice part of the complexity for consistency and gave a perfectly straightforward inverse optics account according to which, as Atherton (this volume) shows in detail, "the visual world is the result of a calculation in which the retinal image serves as a step in the calculation."

\footnotetext{
${ }^{26}$ This view of Descartes was shared by Leibniz, who also argued, in his New Essay Concerning Human Understanding, "that our ideas, even those of sensible things, come from within our own soul."
} 
Cudworth, in his account of perception, followed Descartes very closely.

Nevertheless some aspects of both his emphasis and his framing of the problem are of interest in the present context. He was, in his more general perspective, strongly opposed to Descartes' sharp distinction between the human mind and animal mechanisms. "On Cudworth's view, anything is incorporeal which has a force of its own, anything which is not merely the passive recipient of pushes and pulls. Not everything which is incorporeal is mental, and not everything which is mental is conscious. .. The essential division, as Cudworth sees it, is that between the mechanical and the teleological, not between the unthinking and the thinking. He rejects the Cartesian view that animals are mechanisms, and with it the sharp Cartesian contrast between the animal and the human. What links the human with the animal are instincts: these, Cudworth maintains, are certainly not mechanical, for they have ends, which no mechanism can have, and since they are not mechanical, they cannot be corporeal. .. To Descartes there is simply the dualism of mind and matter; but, as we saw, Cudworth's division of reality comes at a different point, as a dualism of the active and the passive." (Passmore, 1951, p. 23/24)

Cudworth clearly noted that "sense, which either lies in the same level with that particular material object which is perceived, or rather under it and beneath it, cannot emerge to any knowledge or truth concerning it." (1731, p. 95) He therefore argued for a rich "innate cognoscitive power" (ibid., p. 131), by which the mind "is enabled as occasion serves and outward objects invite, gradually and successively to unfold and display it self in a vital manner, by framing intelligible ideas or conceptions within it self of whatsoever hath any entity or cogitability" (ibid., p. 135). Sensible ideas and conceptions of the mind were not passively presented or imprinted by the senses, but rather, "according to nature's instinct, (the mind) hath several seemings or appearances begotten in it of those resisting objects without it at a distance, in respect of colour, magnitude, figure and local motion" (ibid., p. 152). Sensible ideas "are excited and awakened occasionally from the appulse of outward objects knocking at the doors of our senses" (ibid., p. 150). "Sense is but the offering and presenting of some object to the mind, to give it an occasion to exercise its own inward activity upon" (ibid., p. 94). The connection between the "local motions" that the external world exerts on the senses and the ideas and conceptions that were triggered by them in the mind is established "by Nature's law ... though there be no similitude at all betwixt them" (ibid., p. 216). For Cudworth, like for Descartes, sense can be compared to speech by which "Nature as it were talking to us in the sensible objects without, by certain motions as signs from thence communicated to the brain" (ibid., p. 215). As speech is a medium only whose idiosyncratic physical properties bear no direct interest for what is conveyed by it, so the mind, "as by a certain secret instinct, understanding Nature's language, as soon as these local motions are made in the brain, doth not fix its attention immediately upon those motions themselves, as we do not use to do in discourse upon meer speech, but presently exerts such sensible ideas, 
phantasms and cogitations, as Nature hath them to be signs of, whereby it perceives and takes cognizance of many other things both in its own body, and without it, at a distance from it, in order to the good and conservation of it" (ibid., p. 216/7). Similar ideas were expressed by Charles Bonnet (see Yolton, 1984, p. 29).

In our attempts to "better understand how far the passion of the sense reaches, and where the activity of the mind begins", we have to avoid a misconception that still prevails among many perception scientists: "these men not distinguishing betwixt the outward occassion or invitation of those cogitations, and the immediate active or productive cause of them, impute them therefore alike, as well these intelligible, as the other sensible ideas, or phantasms, to the efficiency or activity of the outward objects upon us." (Cudworth, 1731, p. 150)

Cudworth clearly recognized that we have to assume a rich internal structure of the mind in order to account for its active "cognoscitive power"; because of this he played a prominent role in the research programme for cognitive science that Chomsky (1966) set out along historical lines in his Cartesian Linguistics. Cudworth maintained that we cannot understand the active forces of the mind but by assuming "that there are some ideas of the mind which were not stamped or imprinted upon it from the sensible objects without, and therefore must needs arise from the innate vigor and activity of the mind it self" (ibid., p.149), among which he included ideas as contingency, possibility, genus and species, knowledge, justice, verity, equity, obligation, honesty, volition and cogitation, as well as sense itself (ibid., p. 140/149). Furthermore, "there are many relative notions and ideas, attributed as well to corporeal as incorporeal things that proceed wholly from the activity of the mind comparing one thing with another" (ibid., p. 149): The mind "raises and excites within it self the intelligible ideas of cause, effect, means, end, priority and posteriority, equality and inequality, order and proportion, symmetry and asymmetry, aptitude and ineptitude, sign and thing signified, whole and part." (ibid., p. 155)

Reid, whose conception of the perceptual process shared basic elements with the conceptions of Descartes and Berkeley (as to Berkeley, see Hatfield, this volume), emphasized the distinction between 'sensations' and 'perceptions'. ${ }^{27}$ Reid completely divorced 'sensations' that accompany physiological impressions from 'perceptions'. For him perceptions are not mental transformations, supplements or interpretations of sensations. They are rather a completely different kind of mental states, which are characterized by a "strong and irresistable conviction and belief" in the external existence of objects. This belief is "not the effect of reasoning" but part of our biological endowment. "The belief which is implied in it, is the effect of

\footnotetext{
${ }^{27}$ This distinction became a very influential one in perceptual psychology, where it was used, e.g. by Spencer, James, Lotze, Wundt or Helmholtz, in the sense of referring to an alleged hierarchy of processing stages. According to James, for example, sensations and perception "shade gradually into each other, being one and all products of the same psychological machinery of association." (Principles of Psychology, Ch. XIX).
} 
instinct." (An Inquiry into the Human Mind, Ch. VI, § XX). Reid further distinguished "original perceptions" from "acquired perceptions". The "original perceptions" serve as "signs", which elicit "acquired perceptions". The ability to interpret these "signs" was considered by Reid to be innate. By completely cutting any rational relation between sensation and perception, Reid rejected previous ideas of calculation-like processes in perception. Reid thus proposed an extreme version of a sign theory of perception. In his conception the relation between sensations and perceptions cannot be rationally explained; it is simply due to a law of nature. It may be tempting to speculate that Reid would have filled this essential blank in his system if he had been acquainted with evolutionary theory.

Taine, although hardly of interest in his own right in the present context, did give a pithy characterization of how to conceive of perception along such lines (even if he was in general at odds with core ideas of Reid). Taine argued in his framework of naturalistic psychology "that external perception, even when accurate, is an hallucination": "A sensation, and notably a tactile or visual sensation, engenders, by its presence alone, an internal phantom which appears an external object. ... It matters little whether the sensation be purely cerebral and arise spontaneously, without preliminary excitation of the peripheral extremity of the nerve, in the absence of the objects which usually produce the excitation. As soon as ever the sensation is present, the rest follows; the prologue entails the drama. ... If its existence be established by its antecedents, it is confirmed by its consequents." (Taine, 1875, p.1/2).

An elaborated account of a sign theory of perception that is accompanied by an explicit mechanism for explaning how sensations and perceptions are bound together can be found in the work of Helmholtz (e.g. 1878, 1894), who with his sign theory incorporated Kantian elements in his otherwise empiristic stance. According to Helmholtz there is no similarity between our percepts and the structure of the physical environment. The senses provide only signs for our cognitive system, which have to be interpreted according to (ontogenetic or phylogenetic) learning and experience. Unlike pictures, signs do not resemble the object they refer to; however, they mirror lawful relations in the world. However, one has to presuppose a unique functional relation - governed by causal dependencies - between a system of signs and the objects they refer to, i.e. operations on signs correspond to operations on objects. "Innumerable mistakes and incorrect theories in perceptual research were caused by not distinguishing clearly between the concept of a sign and that of a picture." ("Dass man den Begriff des Zeichens und des Bildes bisher in der Lehre von den Wahrnehmungen nicht sorgfältig genug getrennt hat, scheint mir der Grund unzähliger Irrungen und falscher Theorien gewesen zu sein.") (Helmholtz, 1868, p.319). Associative connections mediated by experience allow us to interpret in a correct, i.e. adaptively useful, way the signs which result from the impingement of the outer world on the senses. The sense impressions are thus "signs which we have learnt 
to read; they are a language bestowed upon us by our organization, in which the outer world speaks to us. But we have to learn this language by training and experience, just like our mother language." (Die Sinnesempfindungen sind also "Zeichen, welche wir lesen gelernt haben, sie sind eine durch unsere Organisation uns mitgegebene Sprache, in der die Außendinge zu uns reden; aber diese Sprache müssen wir durch Uebung und Erfahrung verstehen lernen, eben so gut wie unsere Muttersprache." (Helmholtz, 1869, p.393) "The character of these signs is imposed on me by the nature of my sense organs and of my mind; this distinguishes the sign language of perception from the arbitrarily chosen sign of speech and writing." (Die Art der Zeichen ist "mir durch die Natur meiner Sinnesorgane und meines Geistes aufgedrungen. Dadurch unterscheidet sich diese Zeichensprache unserer Vorstellungen von den willkürlich gewählten Lautund Buchstabenzeichen unserer Rede und Schrift" (Helmholtz, 1867, p.446). What we can infer from the external world by this sign language can only be symbolic in character since there is no tertium comperationis. We call our ideas of the external world true when they provide us with sufficient evidence concerning the consequences of our action and when they allow us to infer expected changes correctly (Helmholtz, 1867, p.443).

For Helmholtz the signs themselves exhibit a rich internal structure of operations that mirror corresponding operation on external objects (cf. Hatfield, 1990, p. 208ff.). These ideas provided the foundations for what can be considered an important theoretical insight into the nature of perception, namely the structural or transformational theories of perception. According to these ideas, which are based more or less explicitely on group theoretic concepts, the proper objects of perception are abstract higher-order objects, namely transformational structures. A specific sensory input triggers in sufficiently rich situations an entire transformational structure, which constitutes a 'frame of reference' with respect to which classes of visual 'objects' were defined. The segmentation of the sensory input into 'objects' and 'events' thus depends on the kind of transformational structure that it triggers. Although corresponding intuitions in various forms pervade theoretical ideas on perception since Helmholtz, they were first discussed under the perspective of group theory by Cassirer (1944) and more recently taken up by Shepard (e.g. 1994).

While the insight that the perceptual system imposes a structure on the sensory input that cannot be derived apriori from an analysis of the physical input alone, as well as corresponding notions of triggering, became crucial in ethology (and also in earlier ethology-inspired computational approaches), they have been almost completely ignored within current empiricist accounts, such as sensory data-based computational approaches or data processing models of perception guided by ideas from signal processing and communication engineering. Corresponding insights and intuitions, however, are at the core of the general research programme that has been set out for the study of cognition by Chomsky 
(e.g. 1966, 1996, 2000) and have led, in the domain of language, to real advances in our attempts to develop successful explanatory accounts of the structure of mental representations. 
Acknowledgement. I wish to express my gratitude to Margaret Atherton, Andrea van Doorn, Franz Faul, Don Hoffman and Gary Hatfield for valuable comments on a first draft of this paper.

\section{References}

Albert, M.C. \& Hoffman, D. (1995). Genericity in Spatial Vision. In: R.D. Luce, M. D'Zmura, D. Hoffman, G.J. Iverson \& A.K. Romney (Eds.) Geometrical Representations of Perceptual Phenomena (pp. 95-112). Mahwah, NJ: Lawrence Erlbaum.

Barlow, H. (1961). Possible Principles Underlying the Coding of Sensory Messages (pp.217-234). In W.Rosenblith (Ed.) Sensory Communication. Cambridge, Mass.: MIT Press.

Bennett, B.M., Hoffman, D.D. \& Prakash, C. (1998). Observer Mechanics. A Formal Theory of Perception. San Diego: Academic Press.

Blakemore, R.P. \& Frankel, R.B. (1981). Magnetic Navigation in Bacteria. Scientific American, 245, 42-49.

Boring, E.G. (1921). The Stimulus Error. The American Journal of Psychology, 32, 449471

Buchsbaum, G. \& Gottschalk, A. (1993). Trichromacy, Opponent Colours Coding and Optimum Colour Information Transmission in the Retina. Proceedings of the Royal Society London, B220, 89-113.

Bühler, K. (1922). Die Erscheinungsweisen der Farben. In K. Bühler (Ed.), Handbuch der Psychologie. I.Teil. Die Struktur der Wahrnehmungen (pp. 1-201). Jena: Fischer.

Carey, S. \& Spelke, E.S. (1994). Domain-specific Knowledge and Conceptual Change. In L.A. Hirschfeld \& S.A. Gelman (Eds.), Mapping the Mind. Domain Specifity in Cognition and Culture (pp. 169-200). Cambridge: Cambridge University Press.

Cassirer, E. (1929). Philosophie der symbolischen Formen. Dritter Teil: Phänomenologie der Erkenntnis. Berlin: Bruno Cassirer.

Cassirer, E. (1944). The Concept of Group and the Theory of Perception. Philosophy and Phenomenological Research, 5, 1-35.

Chomsky, N. (1966). Cartesian Linguistics. A Chapter in the History of Rationalist Thought. New York: Harper \& Row.

Chomsky, N. (1996). Power and Prospect. Reflections on Human Nature and the Social Order. London: Pluto Press.

Chomsky, N. (2000). New Horizons in the Study of Language and Mind. Cambridge: Cambridge University Press.

Cudworth, R. (1731). A Treatise Concerning Eternal and Immutable Morality. London: James and John Knapton (Reprinted 1976 by Garland, New York). 
Dretske, F. (1986) Misrepresentation. In R.J. Bogdan (Ed.), Belief: Form, Content, and Function (pp.17-36). Oxford: Oxford University Press.

Fodor, J.A. (1975). The Language of Thought. Cambridge, Mass.: Harvard University Press.

Gallistel, C.R. (1998). Symbolic processes in the brain: the case of insect navigation. In: D. Scarborough \& S. Sternberg (Eds.), Methods, models and conceptual issues. An invitation to cognitive science, Vol. 4. (pp. 1-51) Cambridge, Mass.: MIT Press.

Gelb, A. (1929). Die 'Farbenkonstanz' der Sehdinge. In A. Bethe, G.v. Bergmann, G. Embden, \& A. Ellinger (Eds.), Handbuch der normalen und pathologischen Physiologie. Bd.12, 1.Hälfte. Receptionsorgane II. (pp. 594-678). Berlin: Springer.

Gelb, A. (1932). Die Erscheinungen des simultanen Kontrastes und der Eindruck der Feldbeleuchtung. Zeitschrift für Psychologie, 127, 42-59.

Gilchrist, A.L. (1994). Theories of Lightness Perception. In: A.L. Gilchrist (Ed.), Lightness, Brightness, and Transparency (pp. 1-34). Hillsdale, NJ: Lawrence Erlbaum.

Gombrich, E.H. (1982). The Image and the Eye. Oxford: Phaidon Press.

Hassenstein, B. \& Reichardt, W. (1956). Systemtheoretische Analyse der Zeit, Reihenfolge und Vorzeichenauswertung bei der Bewegungsperzeption des Rüsselkäfers Chlorophanus. Zeitschrift für Naturforschung, 11b, 513-524.

Hatfield, G. (1990). The Natural and the Normative. Theories of Spatial Perception from Kant to Helmholtz. Cambridge, Mass.: MIT Press

Heider, F. (1930). Die Leistungen des Wahrnehmungssystems. Zeitschrift für Psychologie, 114, 371-394.

Helmholtz, H.v. (1855). Über das Sehen des Menschen. In Vorträge und Reden. 4. Aufl., Bd.1, 1896. Braunschweig: Vieweg.

Helmholtz, H.v. (1867). Handbuch der Physiologischen Optik. Hamburg: Voss.

Helmholtz, H.v. (1868). Die neueren Fortschritte in der Theorie des Sehens. In Vorträge und Reden. 4. Aufl., Bd.1, 1896. Braunschweig: Vieweg.

Helmholtz, H.v. (1869). Ueber das Ziel und die Fortschritte der Naturwissenschaft. In Vorträge und Reden. 4. Aufl., Bd.1, 1896.

Braunschweig: Vieweg.

Helmholtz, H.v. (1878). Die Thatsachen in der Wahrnehmung. In Vorträge und Reden. 4. Aufl., Bd.2, 1896. Braunschweig: Vieweg.

Helmholtz, H.v. (1894). Ueber den Ursprung der richtigen Deutung unserer Sinneseindrücke. Zeitschrift für Psychologie und Physiologie der Sinnesorgane, 7, 81-96.

Hering, E. (1920). Grundzüge der Lehre vom Lichtsinn. Berlin: Springer.

Hochberg, J. (1980). Pictorial Function and Perceptual Structure. In: M.A. Hagen (Ed.), The Perception of Pictures, Vol. II (pp. 47-93). New York: Academic Press. 
Hoffman, D.D. (1996). What Do We Mean by "Structure of the World"? In D.C. Knill \& W. Richards (Eds.), Perception as Bayesian Inference (pp. 219-221). New York: Cambridge University Press.

Kardos, L. (1934). Ding und Schatten. Eine experimentelle Untersuchung über die Grundlagen des Farbensehens. Leipzig: Barth.

Knill, D.C., Kersten D. \& Mamassian, P. (1996). Implications of a Bayesian Formulation of Visual Information for Processing for Psychophysics. In D.C. Knill \& W. Richards (Eds.), Perception as Bayesian Inference (pp. 239286). New York: Cambridge University Press.

Knill, D.C. \& Richards W. (Eds.), Perception as Bayesian Inference. New York: Cambridge University Press.

Kemp, M. (1990). The Science of Art. Optical Themes in Western Art from Brunelleschi to Seurat. New Haven, CT: Yale University Press.

Koenderink, J.J. (1998). Pictorial Relief. Philosophical Transactions of the Royal Society London, A 356, 1071-1086.

Koenderink, J.J. \& van Doorn, A.J. (1998). Exocentric Pointing. In: L.R. Harris \& M. Jenkin (eds.), Vision and Action (pp. 295-313). Cambridge: Cambridge University Press.

Koenderink, J.J., van Doorn, A.J. \& Kappers, A. (2000). Surfaces in the Mind's Eye. In: R. Cipolla \& R. Martin (eds.), The Mathematics of Surfaces IX (pp. 180-193. London: Springer.

Koffka, K. (1932). Some Remarks on the Theory of Colour Constancy. Psychologische Forschung, 16, 329-354.

Landau, B., Spelke, E.S. \& Gleitman H. (1984). Spatial Knowledge in a Young Blind Child. Cognition, 16, 225-260.

Lewontin, R.C. (1998). The Evolution of Cognition: Questions We Will Never Answer. In D. Scarborough \& S. Sternberg (Eds.), An Invitation to Cognitive Science: Methods, Models, and Conceptual Issues (pp. 107-132). Cambridge, Mass.: MIT Press.

Lindberg, D.C. (1976). Theories of Vision form Al-Kindi to Kepler. Chicago: University of Chicago Press.

MacLeod, R.B. (1932). An Experimental Investigation of Brightness Constancy. Archives of Psychology, 135, 5-102.

MacLeod, D.I.A. \& Willen, J.D. (1995). Is there a Visual Space? In: R.D. Luce, M. D'Zmura, D. Hoffman, G.J. Iverson \& A.K. Romney (Eds.) Geometrical Representations of Perceptual Phenomena (pp. 47-60). Mahwah, NJ: Lawrence Erlbaum.

Mausfeld, R. (1998). Color Perception: From Grassmann Codes to a Dual Code for Object and Illumination Colors. In: W. Backhaus, R. Kliegl, J. Werner (Eds.), Color Vision (pp. 219-250). De Gruyter, Berlin/New York.

Mausfeld, R. (2003). 'Colour' as part of the format of two different perceptual primitives: The dual coding of colour. In: R. Mausfeld \& D. Heyer (Eds.), Colour Perception: Mind and the Physical World. Oxford: Oxford University Press. 
Mausfeld, R. (2002). Conjoint Representations and the Mental Capacity for Multiple Simultaneous Perspectives. In M. Atherton, H. Hecht, \& B. Schwartz (Eds.), Looking into Pictures: An Interdisciplinary Approach to Pictorial Space (pp. 17-60). Cambridge, Mass.: MIT Press.

Mausfeld, R. \& Niederée, R. (1993). Inquiries into Relational Concepts of Colour Based on an Incremental Principle of Colour Coding for Minimal Relational Stimuli. Perception, 22, 427-462.

Mausfeld, R. \& Andres, J. (2002). Second Order Statistics of Colour Codes Modulate Transformations that Effectuate Varying Degrees of Scene Invariance and Illumination Invariance, Perception, 31, 209-224.

Meltzoff, A.N. (1995). Understanding the intentions of others: Re-enactment of intended acts by 18-month-old children. Developmental Psychology, 31, 838850.

Michotte, A. (1946). La perception de la causalité. Louvain: Fondation Universitaire de Belgique.

Michotte, A, (1948). L'énigma psychologique de la perspective dans le dessin linéaire. Bulletin des Lettres de l'Académie Royale de Belgique, 34, 268-288.

Nakayama, K., He, Z.J. \& Shimojo, S. (1995). Visual Surface Representation: A Critical Link between Lower-Level and Higher-Level Vision. In: S.M. Kosslyn \& D.N. Osherson (Eds.), Visual Cognition. An Invitation to Cognitive Sciences. Vol 2. (pp. 1-70). Cambridge, Mass.: MIT Press.

Passmore, J.A. (1951). Ralph Cudworth: An Interpretation. Cambridge: Cambridge University Press.

Poggio, T. (1990). Vision: The 'Other' Face of AI. In K.A. Mohyeldin Said, W.H. Newton-Smith, R. Viale \& K.V. Wilkes, (Eds.) Modelling the Mind (pp. 139154). Oxford: Clarendon Press.

Premack, D. \& Premack, A.J. (1995). Intention as Psychological Cause. In: D. Sperber, D. Premack \& A.J. Premack (Eds.), Causal Cognition (pp. 185-199). Oxford: Clarendon.

Richards, W. (1986). Priors by Design. In D.C. Knill \& W. Richards (Eds.), Perception as Bayesian Inference (pp. 225-228). New York: Cambridge University Press.

Rock, I. (1983). The Logic of Perception. Cambridge, Mass.: MIT Press.

Rodgers, S. (1995). Perceiving Pictorial Space. In: W. Epstein \& S. Rogers (Eds.), Perception of Space and Motion (pp. 119-163). San Diego: Academic Press.

Sabra, A.I. (1989). The Optics of Ibn Al-Haytham. Books I-III. On Direct Vision. Translation and Commentary. 2 Vol. London: The Warburg Institute.

Scholl, B.J. \& Tremoulet, P.D. (2000). Perceptual Causality and Animacy. Trends in Cognitive Science, 4, 299-309.

Schöne, W. (1954). Über das Licht in der Malerei. Berlin: Gebr. Mann.

Sedgwick, H.A. (1986). Space Perception. In: K.R. Boff, L. Kaufman \& J.P. Thomas (eds.), Handbook of Perception and Human Performance. Vol.I. Sensory Processes and Perception. New York: Wiley. 
Shepard, R.N. (1987). Evolution of a Mesh Between Principles of the World and Regularities of the World. In J. Dupré (Ed.), The Latest on the Best. Essays on Evolution and Optimality (pp. 251-275), Cambridge, Mass.: Bradford.

Shepard, R.N. (1994). Perceptual-Cognitive Universals as Reflections of the World. Psychonomic Bulletin \& Review, 1, 2-28.

Smith, B. \& Casati, R. (1994). Naive Physics. Philosophical Psychology, 7, 227-247. Spelke, E.S. (1995). Initial knowledge: Six Suggestions. In: J. Mehler \& S. Franck (Eds.), Cognition on Cognition (pp. 433-447). Cambridge, Mass.: MIT Press. Streri, A., Spelke, E.S. \& Rameix, E. (1993). Modality-Specific and Amodal Aspects of Object Perception in Infancy: The Case of Active Touch. Cognition, 47, 251-279.

Taine, H. (1875). On Intelligence. Vol. II. New York: Henry Holt.

Tolman, E.C. \& Brunswik, E. (1935). The Organism and the Causal Texture of the Environment. Psychological Review, 42, 43-77.

Trevarthen, C. (1998). The Concept and Foundations of Infant Intersubjectivity. In: Bråten, S. (Ed.) Intersubjective Communication and Emotion in Early Ontogeny (pp. 15-46). Cambridge: Cambridge University Press.

von Szily, A. (1921). Stereoskopische Versuche mit Schattenrissen. Gräfes Archiv für Ophtalmologie, 105, 964-972.

Yolton, J.W. (1984). Perceptual Acquaintance from Descartes to Reid. Minneapolis: University of Minnesota Press. 